\title{
COMMON RANGE OF CO-ANALYTIC TOEPLITZ OPERATORS
}

\author{
JOHN E. MC CARTHY
}

\section{INTRODUCTION}

A weight on the unit circle $\mathbb{T}$ is a positive function in $L^{1}(d \theta)$, where $d \theta$ denotes Lebesgue measure, and we shall use $\mathscr{W}$ for the set of weights $w$ for which $\int_{\mathbb{T}} \log \left(w\left(e^{i \theta}\right)\right) d \theta>-\infty . H^{2}(w)$ will denote the closure of the (analytic) polynomials in $L^{2}(w d \theta)$. The Smirnov Class $N^{+}$can be defined to be

$$
N^{+}=\bigcup_{w \in \mathscr{W}} H^{2}(w)
$$

(This is not the usual definition-see $\S 1$.)

In [He] Helson observed that this representation of $N^{+}$as a union of Hilbert spaces enables one to define a locally convex inductive limit topology (where a neighborhood base for zero is given by those absolutely convex sets whose intersection with every $H^{2}(w)$ is a neighborhood of zero; for basic facts about inductive limits see [Kö] or [Wi]). We shall call this topology the Helson topol$o g y$, and denote it by $H$. A linear functional $f$ on $\left(N^{+}, H\right)$ is continuous if and only if its restriction to each $H^{2}(w)$ is continuous. In particular, $f$ is a continuous linear functional on $H^{2}$, so it can be considered an $H^{2}$ function in its own right. The following theorem is from [He].

Theorem 0.1 [Helson]. The following are equivalent

(i) $f$ in $H^{2}$ defines a continuous linear functional on $\left(N^{+}, H\right)$;

(ii) For each $w$ in $\mathscr{W}$ there is a function $k$ in $H_{0}^{1}$ such that $\int|f+\bar{k}|^{2} \frac{1}{w}<$ $\infty$;

(iii) For every $h$ in $H^{2}$, the positive Fourier coefficients of $f$ can be represented by $\hat{f}(n)=\left(k, S^{n} h\right)$ for some $k$ in $H^{2}$;

(iv) For each $h$ in $H^{\infty}, f$ is in the range of the Toeplitz operator $T_{\bar{h}}$.

A desire to characterize those functions satisfying (iv) first aroused our interest in the topologies on $N^{+}$.

Received by the editors November 14, 1989 and, in revised form, May 15, 1990.

1980 Mathematics Subject Classification (1985 Revision). Primary 47B35, 46E20; Secondary 30D55.

(C) 1990 American Mathematical Society 
N. Yanagihara [Ya] studied the dual of $N^{+}$in a metric topology, which is complete but not locally convex, given by the metric

$$
\rho(f, g)=\int_{\mathbb{T}} \log (1+|f-g|) d \theta .
$$

He showed that a linear functional $\Lambda: z^{k} \mapsto \lambda_{k}$ is continuous if and only if $\lambda_{k}=O(\exp (-c \sqrt{k}))$ for some positive constant $c$. There is a simple proof of this result in [ $\left.\mathrm{M}^{\mathrm{C}} \mathrm{C} 1\right]$. In $\S 2$, we show that $\left(N^{+}, H\right)^{*}=\left(N^{+}, \rho\right)^{*}$, and thus solve our original problem, viz. a function $f$ is in the range of every co-analytic Toeplitz operator if and only if $\hat{f}(k)=O\left(e^{-c \sqrt{k}}\right)$ for some positive constant $c$ (Theorem 2.2).

\section{Preliminaries}

The normal way of defining the Smirnov class is as follows: first, one defines the Nevanlinna class $N$ as the set of analytic functions $f$ on the unit disk $\mathbb{D}$ for which the function $\log ^{+}|f(z)|$ has a harmonic majorant. Functions in this class have nontangential limits almost everywhere, and so as usual we shall think of them either as functions on the disk or on the circle, as convenient. The Smirnov class $N^{+}$then consists of those functions $f$ in $N$ for which

$$
\lim _{r \rightarrow 1} \int_{\mathbb{T}} \log ^{+}\left|f\left(r e^{i \theta}\right)\right| d \theta=\int_{\mathbb{T}} \log ^{+}\left|f\left(e^{i \theta}\right)\right| d \theta .
$$

For basic facts about these classes, see, for example, [Ga] or [Du].

It is an old theorem of the brothers Nevanlinna [Ne] that $N^{+}$is precisely the set $\left\{g / h: g, h \in H^{\infty}, h\right.$ outer $\}$. This latter set is the same if the stipulation that $g$ and $h$ be in $H^{\infty}$, the space of bounded analytic functions on the disk, is replaced by the requirement that they be in $H^{2}$, the classical Hardy space of square-integrable functions on the circle with vanishing negative Fourier coefficients. That this seemingly larger set of quotients is, in fact, the same can be seen as follows (the argument is from [Ni]): Suppose $g$ and $h$ are in $H^{2}$, $h$ outer. Let $g=I G$ be the inner-outer factoring of $g$. Let $G_{e}$ be the outer function with modulus $\min (1,|G|), h_{e}$ defined similarly. Put $g_{1}=I G_{e} h_{e} / h$, and $h_{1}=G_{e} h_{e} / G$. Then $g_{1}$ and $h_{1}$ are in $H^{\infty}, h_{1}$ is outer, and their quotient is $g / h$.

For a fixed outer function $h$, a function $f$ is in $H^{2}\left(|h|^{2}\right.$ ) (the closure of the polynomials in $L^{2}\left(\left|h\left(e^{i \theta}\right)\right|^{2} d \theta\right)$ if and only if, for any $\varepsilon>0$, there exists a polynomial $p$ such that

$$
\int_{\mathbb{T}}|f-p|^{2}|h|^{2} d \theta<\varepsilon
$$

This is the same as saying that $f h$ is in the $L^{2}(d \theta)$-closure of $\{p h: p$ a polynomial ; because $h$ is outer, the closure of this last set is all of $H^{2}$, so $f h$ is in $H^{2}$. Therefore $H^{2}\left(|h|^{2}\right)$ is the same as $H^{2} / h$, so

$$
N^{+}=\bigcup_{h \in H^{2}} H^{2}\left(|h|^{2}\right) \text {. }
$$


By the well-known theorem of Szegö, $\left\{|h|^{2}: h \in H^{2}\right\}=\{w: w \in \mathscr{W}\}$ (which in turn $=\left\{w: H^{2}(w) \neq L^{2}(w)\right\}[\mathrm{Co}]$ ), so we see that our original definition of $N^{+}$agrees with the classical one.

Let $P$ be the orthogonal projection from $L^{2}(d \theta)$ onto $H^{2}$. Then for any function $m$ in $L^{\infty}(d \theta)$ the Toeplitz operator with symbol $m, T_{m}$, is defined as that linear operator on $H^{2}$ that sends $f$ to $P m f$. If $m$ is the complex conjugate of a function in $H^{\infty}, T_{m}$ is called a co-analytic Toeplitz operator.

For completeness, we will now give a proof of the equivalence of (i) and (iv) in Theorem 0.1.

First observe that if a weight $w$ is decreased, the space $H^{2}(w)$ increases, and its dual decreases. So the dual of $\left(N^{+}, H\right)$ is the same as the intersection of the duals of the $H^{2}(w)$ where $w$ ranges, not over all of $\mathscr{W}$, but only over those weights that also satisfy $\|w\|_{\infty} \leq 1$. So a function $f$ is in $\left(N^{+}, H\right)^{*}$ if and only if, for each outer function $h$ with $\|h\|_{\infty} \leq 1$, there exists a constant $C$ such that for all polynomials $p$

$$
\left|\int_{\mathbb{T}} p \bar{f} d \theta\right|^{2} \leq C \int_{\mathbb{T}}|p|^{2}|h|^{2} d \theta .
$$

This is true if and only if there exists a $g$ in $H^{2}\left(|h|^{2}\right)$ of norm less than or equal to $\sqrt{C}$ for which

$$
\int_{\mathbb{T}} p \bar{f} d \theta=\int_{\mathbb{T}} p \bar{g}|h|^{2} d \theta .
$$

But as we observed earlier $g$ can then be written as $k / h$ for some $k$ in $H^{2}$, which means that

$$
\int_{\mathbb{T}} p \bar{f} d \theta=\int_{\mathbb{T}} p \bar{k} h d \theta
$$

for all $p$; that is, $f=P \bar{h} k=T_{\bar{h}} k$. Remarking that for any inner function $I$, $T_{\overline{I h}} I k=T_{\bar{h}} k$, we have proved that $f$ is in the dual of $\left(N^{+}, H\right)$ if and only if it is in the range of all Toeplitz operators with co-analytic symbol.

\section{THE DUAL OF $\left(N^{+}, H\right)$}

Let $\rho$ be the metric on $N^{+}$given by $\rho(f, g)=\int_{\mathbb{T}} \log (1+|f-g|) d \theta$, mentioned in the introduction. The dual of $N^{+}$is the same in both the $H$ and $\rho$ topologies.

Theorem 2.1. $\left(N^{+}, H\right)^{*}=\left(N^{+}, \rho\right)^{*}$.

Proof. The proof is in two stages: first, we show that each $H^{2}(w)$ embeds continuously in $\left(N^{+}, \rho\right)$, so $\left(N^{+}, \rho\right)^{*}$ is contained in each $H^{2}(w)^{*}$, and likewise in their intersection, which is just $\left(N^{+}, H\right)^{*}$. Then we show that $\left(N^{+}, \rho\right)$ embeds in $\left(N^{+}, H\right)$, so their duals actually coincide.

(i) Let us show that the inclusion map from $H^{2}\left(|h|^{2}\right)$ into $\left(N^{+}, \rho\right)$ is continuous for every outer function $h$. By first embedding $H^{2}\left(|h|^{2}\right)$ into $H^{2}\left(\left|h_{e}\right|^{2}\right)$, 
if necessary, we can assume that $\|h\|_{\infty} \leq 1$. So suppose that some sequence $f_{n}$ tends to zero in $H^{2}\left(|h|^{2}\right)$, and let $\varepsilon>0$. For $n$ sufficiently large,

$$
\int_{\mathbb{T}}\left|f_{n}\right|^{2}|h|^{2}<\varepsilon^{2} \inf _{|E|=\varepsilon} \int_{E}|h|^{2} d \theta
$$

and then $\left|f_{n}\right|$ is less than $\varepsilon$ except on a set of measure $\varepsilon$. So for fixed large $n$ we can write $\mathbb{T}=E_{1} \cup E_{2}$, where $\log \left(1+\left|f_{n}\right|\right)$ is less than $\varepsilon$ on $E_{1}$, and $\left|E_{2}\right|<\varepsilon$

$$
\begin{aligned}
\int_{E_{2}} \log \left(1+\left|f_{n}\right|\right) d \theta & =\int_{E_{2}} \log \left(|h|+\left|h f_{n}\right|\right) d \theta-\int_{E_{2}} \log |h| d \theta \\
& \leq \int_{E_{2}} \log \left(1+\left|h f_{n}\right|\right) d \theta-\int_{E_{2}} \log |h| d \theta
\end{aligned}
$$

and applying the inequality $\log (1+x)<1+x^{2}$ to the first term on the right-hand side, we get

$$
\int_{E_{2}} \log \left(1+\left|f_{n}\right|\right) d \theta<\left|E_{2}\right|+\int_{E_{2}}\left|h f_{n}\right|^{2} d \theta-\int_{E_{2}} \log |h| d \theta .
$$

Thus we get that for all $n$ sufficiently large

$$
\int_{\mathbb{T}} \log \left(1+\left|f_{n}\right|\right) d \theta<(2 \pi+1) \varepsilon+\int_{\mathbb{T}}\left|h f_{n}\right|^{2} d \theta-\sup _{\left|E_{2}\right| \leq \varepsilon} \int_{E_{2}} \log |h| d \theta .
$$

Let $n$ tend to infinity, and we get

$$
\limsup _{n \rightarrow \infty} \int_{\mathbb{T}} \log \left(1+\left|f_{n}\right|\right) d \theta \leq(2 \pi+1) \varepsilon-\sup _{\left|E_{2}\right| \leq \varepsilon} \int_{E_{2}} \log |h| d \theta .
$$

Now let $\varepsilon$ tend to zero, and the right-hand side tends to zero, because $\log |h|$ is integrable, so

$$
\int_{\mathbb{T}} \log \left(1+\left|f_{n}\right|\right) d \theta \rightarrow 0
$$

and the embedding into $\left(N^{+}, \rho\right)$ is continuous.

Therefore $\left(N^{+}, \rho\right)^{*}$ is contained in each $H^{2}\left(|h|^{2}\right)^{*}$, so $\left(N^{+}, \rho\right)^{*} \subseteq\left(N^{+}, H\right)^{*}$.

(ii) Now we will show that the identity mapping from $\left(N^{+}, \rho\right)$ to $\left(N^{+}, H\right)$ is continuous.

Suppose $f_{n}$ tends to zero in $\left(N^{+}, \rho\right)$. It is sufficient to prove that a subsequence tends to zero in $\left(N^{+}, H\right)$, so we can assume without loss of generality that $\int_{\mathbb{T}} \log \left(1+\left|f_{n}\right|\right)<2^{-n}$, and that $f_{n}$ converges to zero pointwise almost everywhere. Let $w=1+\sum_{n=0}^{\infty}\left|f_{n}\right|$. Then

$$
\begin{aligned}
\log (w) & =\log \left(1+\sum_{n=0}^{\infty}\left|f_{n}\right|\right) \\
& \leq \log \prod_{n=0}^{\infty}\left(1+\left|f_{n}\right|\right) \\
& =\sum_{n=0}^{\infty} \log \left(1+\left|f_{n}\right|\right),
\end{aligned}
$$


so $\log (w)$ is integrable. Therefore there exists an outer function $h$ in $N^{+}$with $|h|=w$ almost everywhere. $h$ is given by

$$
h(z)=\exp \left(\int_{\mathbb{T}} \frac{e^{i \theta}+z}{e^{i \theta}-z} w\left(e^{i \theta}\right) d \theta\right),
$$

which is in the Nevanlinna class because $\log ^{+}|h|$ has the Poisson integral of $w$ as a harmonic majorant, and is in the Smirnov class because it is outer-see [Ga, p. 71]. By the Nevanlinnas' theorem, we can write $h=h_{1} / h_{2}$, with $h_{1}$ and $h_{2}$ in $H^{\infty}$. Now

$$
1+\sum_{n=0}^{\infty}\left|f_{n}\right|=\frac{h_{1}}{h_{2}}
$$

so

$$
\left|h_{2}\right|\left|f_{n}\right| \leq\left|h_{1}\right|
$$

for each $n$. Therefore by Lebesgue's dominated convergence theorem, $\int_{\mathbb{T}}\left|f_{n}\right|^{2}\left|h_{2}\right|^{2} d \theta$ tends to zero.

But if $f_{n}$ tends to zero in $H^{2}\left(|h|^{2}\right)$, it must tend to zero in $\left(N^{+}, H\right)$, because any neighborhood of zero in the latter, $U$, contains a neighborhood of zero in the former, as a result $f_{n}$ must eventually be in $U$.

Therefore $H$ is a weaker topology than $\rho$, so $\left(N^{+}, H\right)^{*}$ is contained in $\left(N^{+}, \rho\right)^{*}$. By part (i), we conclude that the two duals coincide.

We actually proved the following in Theorem 2.1: Let $I$ be the not locally convex inductive limit topology on

$$
N^{+}=\bigcup_{w \in \mathscr{W}} H^{2}(w),
$$

where a neighborhood of zero is any set whose intersection with every $H^{2}(w)$ is a neighborhood of zero. Part (i) of the proof then shows that the inclusion map from $\left(N^{+}, I\right)$ to $\left(N^{+}, \rho\right)$ is continuous, because the pull-back of any open set is open in each $H^{2}(w)$; part (ii) shows the inclusion map from $\left(N^{+}, \rho\right)$ to $\left(N^{+}, I\right)$ is continuous, so $\rho$ and $I$ are equivalent topologies. As $H$ is the finest locally convex topology coarser than $I,\left(N^{+}, H\right)$ and $\left(N^{+}, I\right)$ must have the same duals.

In $\left[\mathrm{M}^{\mathrm{C}} \mathrm{C} 1\right]$ it is shown that the Helson topology is actually metrizable, and that in it, the Taylor series of every function in $\mathrm{N}^{+}$converges to that function; although, for example, the series $\sum_{n=0}^{\infty} z^{n}$ does not converge to $1 /(1-z)$ in either $\left(N^{+}, \rho\right)$ or $H^{2}\left(|1-z|^{2}\right)$, or indeed any space $H^{2}(w)$.

Combining the characterization of $\left(N^{+}, H\right)^{*}$ from Theorem 0.1, and Yanagihara's description of $\left(N^{+}, \rho\right)^{*}$ mentioned in the introduction, we get our most important result as a corollary of 2.1 :

Theorem 2.2. The function $f$ is in the range of every nonzero co-analytic Toeplitz operator if and only if there exists a constant $c>0$ such that $\hat{f}(n)=$ $O\left(e^{-c \sqrt{n}}\right)$. 


\section{QUESTIONS}

It is easy to define Toeplitz-like operators, multiplication followed by projection, in many other settings, and one can ask what the analogue of Theorem 2.2 might be. There are two particular cases of interest:

(i) Let $\mu$ be a compactly supported measure on $\mathbb{C}$, and let $P^{2}(\mu)$ be the closure of the polynomials in $L^{2}(\mu)$. Assume, moreover, that $P^{2}(\mu) \neq L^{2}(\mu)$, to avoid degeneracy. Call a Toeplitz operator co-analytic if its symbol is the complex conjugate of a function in $P^{2}(\mu) \cap L^{\infty}(\mu)$. What can be said about the common range of all co-analytic Toeplitz operators? If $\mu$ is area measure on the disk, Theorem 2.2 holds exactly as stated $\left[\mathrm{M}^{\mathrm{C}} \mathrm{C} 2\right]$, but what happens for more complicated measures?

(ii) Consider the case of the unit ball in $\mathbb{C}^{n}$. M. Nawrocki has characterised the dual of the Smirnov class as those linear functionals $\Lambda$ such that $\Lambda\left(z^{\alpha}\right)=$ $O\left(\exp \left(-c|\alpha|^{n /(n+1)}\right)\right)[\mathrm{Na}]$. The above arguments yield that this is a sufficient decay condition for a function to be in the range of every co-analytic Toeplitz operator on the sphere; but the necessity argument breaks down because

$$
\bigcup_{h \in H^{\infty}\left(B_{n}\right)} H^{2}\left(|h|^{2}\right)
$$

is strictly smaller than the Smirnov class. So what are necessary and sufficient conditions in this case?

\section{ACKNOWLEDGMENT}

The author would like to thank Mark Davis and Henry Helson for valuable conversations.

\section{REFERENCES}

[Co] J. B. Conway, Subnormal operators, Pitman, Boston, 1981.

[Du] P. L. Duren, Theory of $H^{p}$ spaces, Academic Press, New York, 1970.

[Ga] J. B. Garnett, Bounded analytic functions, Academic Press, New York, 1981.

[He] H. Helson, Large analytic functions II, in Analysis and Partial Differential Equations (Cora Sadosky, ed.), Marcel Dekker, Basel, 1990.

[Kö] G. Köthe, Topological vector spaces I, Springer-Verlag, Heidelberg, 1969; Topological vector spaces II, Springer-Verlag, Heidelberg, 1979.

$\left[\mathrm{M}^{\mathrm{C}} \mathrm{C} 1\right]$ J. E. $\mathrm{M}^{\mathrm{C}}$ Carthy, Topologies on the Smirnov class (to appear).

$\left[\mathrm{M}^{\mathrm{C}} \mathrm{C} 2\right] \ldots$ Weighted Bergman spaces, Proceedings of a Conference on Function Spaces (Edwardsville, 1990) (to appear).

[Na] M. Nawrocki, Linear functionals on the Smirnov class of the unit ball in $\mathbb{C}^{n}$. Ann. Acad. Sci. Fenn. Ser. A I Math. 14 (1989), 369-379.

[Ne] F. Nevanlinna and R. Nevanlinna, Eigenschaften analytischer Funktionen in der Umgebung einer singulären Stelle oder Linie, Acta Soc. Sci. Fenn. (5) 50 (1922).

[Ni] N. K. Nikolskii, Treatise on the shift operator, Springer-Verlag, Berlin, 1985. 
[Wi] A. Wilansky, Modern methods in topological vector spaces, McGraw-Hill, New York, 1978.

[Ya] N. Yanagihara, Multipliers and linear functionals for the class $N^{+}$, Trans. Amer. Math. Soc. 180 (1973), 449-461.

ABSTRACT. We characterise the intersection of the ranges of all co-analytic Toeplitz operators, by considering this set as the dual space of the Smirnov class in a certain topology.

Department of Mathematics, Indiana University, Bloomington, Indiana 47405 